\section{MR Imaging Findings in Intracranial Dural Arteriovenous Fistula Shunt with Retrograde Cortical Venous Drainage Using Susceptibility-Weighted Angiography}

We read with special interest the article of Noguchi et al entitled "Intracranial Dural Arteriovenous Fistula with Retrograde Cortical Venous Drainage: Use of Susceptibility-Weighted Imaging in Combination with Dynamic Susceptibility Contrast Imaging." The authors reported the use of SWI in combination with DSC for the imaging of related retrograde cortical venous drainage (RCVD) in patients with intracranial dural arteriovenous fistula (DAVF). They suggested that SWI in combination with DSC could be used to characterize the presence of RCVD in patients with DAVFs showing dilated cerebral veins on the surface of the brain. In this article, the RCVD was imaged by using minimal intensity projection (mIP) views.

Susceptibility-weighted angiography (SWAN) is a new 3D T2*based gradient-echo sequence generating several echoes that are read out at different TE times. ${ }^{2}$ During each TR, the SWAN sequence captures multiple TE readouts: short TEs provide a time-of-flight (TOF) effect, whereas long TEs are responsible for the magnetic-susceptibility effect. Indeed, the SWAN sequence allows high-resolution visualization of both cerebral veins and arteries. By use of either mIP or maximum intensity projection (MIP) reconstructions, the hyperintense arteries can be distinguished from the hypointense veins. ${ }^{2} \mathrm{We}$ report 2 cases of intracranial DAVFs with RCVD explored at 3T (Discovery MR 750; GE Healthcare, Milwaukee, Wisconsin) by using the SWAN sequence. The first case is a 32-year-old man with a right parietal DAVF supplied by the right middle meningeal artery (patient 1, Cognard classification type III). With the SWAN sequence, the RCVD is clearly identified with dilated parietal veins that appeared hyperintense (Fig 1). A 4D dynamic MR angiography (Time-Resolved Imaging of Contrast Kinetics [TRICKS]) showed the DAVF and the related RCVD with an abnormal early arterial filling of the
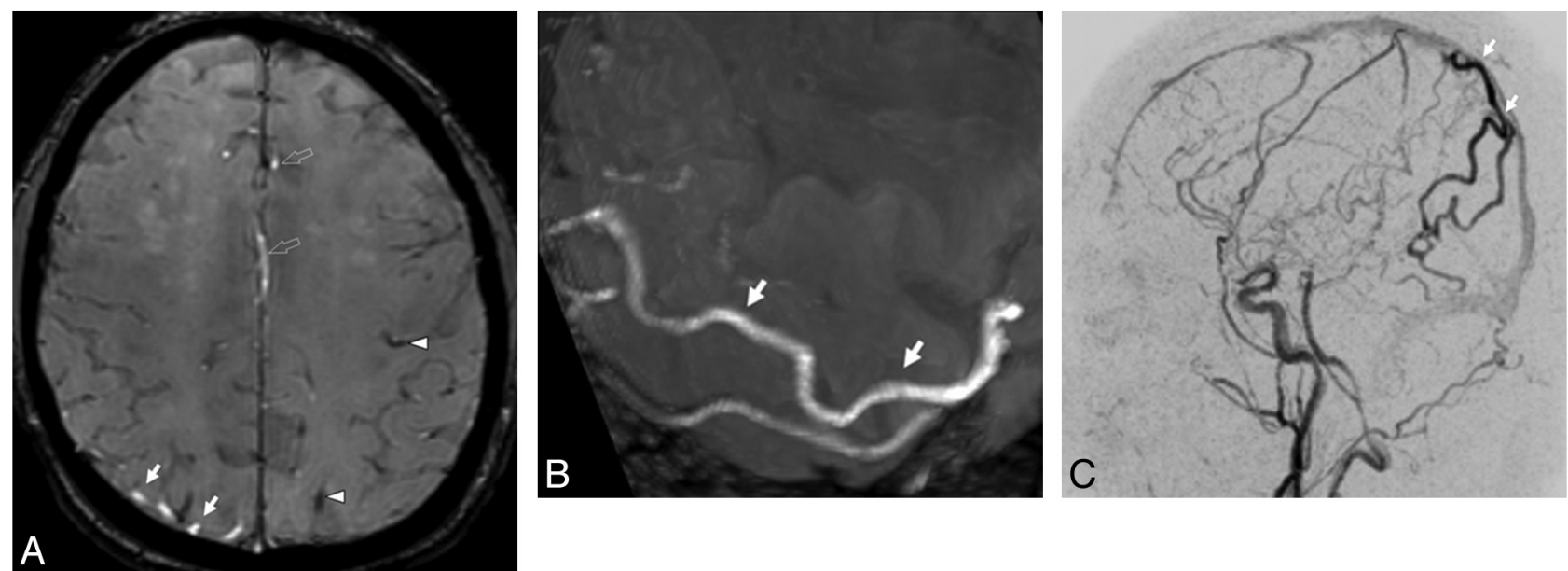

Fig 1. A, A 32-year-old man with a right parietal DAVF supplied by the right middle meningeal artery with venous drainage directly into the cortical veins (Cognard classification type III). On the native axial image of the SWAN sequence (A), brain arteries appeared bright (open arrows), whereas normal cerebral vein signal intensity was decreased (arrowheads). The SWAN sequence also revealed abnormal hyperintense parietal veins (arrows). B, A 32-year-old man with a right parietal DAVF supplied by the right middle meningeal artery with venous drainage directly into cortical veins (Cognard classification type III). With an oblique MIP reformation (B), this retrograde cortical venous drainage was easily detected. $C$, A 32-year-old man with a right parietal DAVF supplied by the right middle meningeal artery with venous drainage directly into the cortical veins (Cognard classification type III). The Sagittal MIP view of the 4D dynamic MR angiography $(C)$ confirmed the early arterial phase filling of the abnormal parietal veins (arrows).
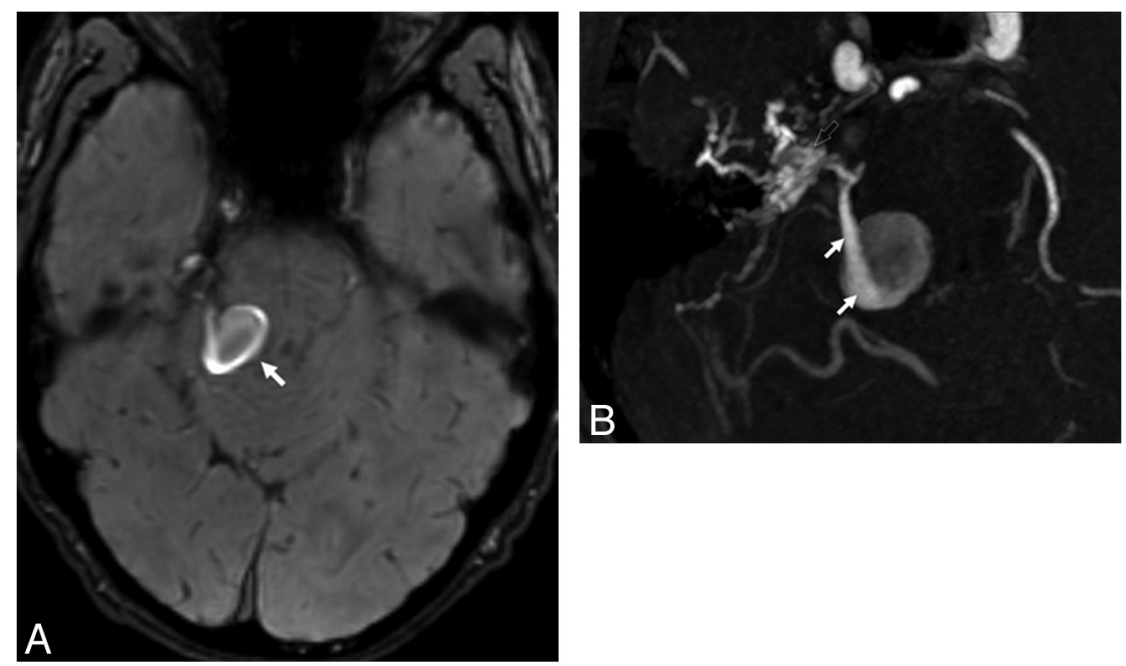

Fig 2. A, A 25-year-old man with a right petrous DAVF supplied by the right middle meningeal artery with retrograde cortical venous drainage and venous ectasia (Cognard classification type IV). On the native axial image of the SWAN sequence (A), the venous ectasia related to the right petrous DAVF appeared in hypersignal (arrow). $B$, A 25-year-old man with a right petrous DAVF supplied by the right middle meningeal artery with retrograde cortical venous drainage and venous ectasia (Cognard classification type IV). With the oblique MIP reformation of a TOF sequence $(B)$, the petrous DAVF was visible (open arrow) as well as the venous ectasia (arrow). Note the TOF-related increased signal intensity due to the increased blood flow through the venous ectasia. 
parietal veins, which appeared bright on the SWAN images (Fig 1). The second case is a 25-year-old man with a right petrous DAVF supplied by the right middle meningeal artery with RCVD and venous ectasia (patient 2, Cognard classification type IV). With the SWAN sequence, both the RCVD and the venous ectasia appeared hyperintense (Fig 2). In this patient, a TOF sequence revealed a marked TOF effect through the venous ectasia related to an increased blood flow (Fig 2).

In the study of Noguchi et al, ${ }^{1}$ typical RCVD appeared in hyposignal on mIP reformations except for 1 patient with a type III DAVF. In this patient, mIP reformations of the SWI sequence failed to detect nondeoxygenated RCVD because in such cases, the arterial blood drained directly into the cortical veins. The typical hypointense pattern of RCVD described by Noguchi et al could be explained by the absence of TOF effect by using the SWI sequence. Indeed, the SWAN sequence differs from the SWI because of the short TEs, which provide a TOF effect within the images.

Our cases suggested that the venous drainage appeared hyperintense by using the SWAN sequence in either type III or IV DAVF and could be easily identified with MIP reconstructions. Moreover, considering the results obtained with SWI and SWAN sequences, we could speculate that the hyperintensity of the RCVD observed in our patients may be more related to a TOF effect than the presence of nondeoxygenated blood within the veins. Our findings indicate the potential value of the SWAN sequence for the diagnosis of intracranial DAVF. Indeed, the visualization of a venous hyperintensity on SWAN images may suggest a related intracranial arteriovenous shunt. Further studies are required to confirm this hypothesis, particularly concerning a DAVF with small and/or very slow-flowing venous drainage.

\section{References}

1. Noguchi K, Kuwayama N, Kubo M, et al. Intracranial dural arteriovenous fistula with retrograde cortical venous drainage: use of susceptibilityweighted imaging in combination with dynamic susceptibility contrast imaging. AJNR Am J Neuroradiol 2010;31:1903-10

2. Boeckh-Behrens T, Lutz J, Lummel N, et al. Susceptibility-weighted angiography (SWAN) of cerebral veins and arteries compared to TOF-MRA. Eur J Radiol 2011 Apr 3. [Epub ahead of print]

J. Hodel

S. Gerber

M. Zins

Department of Radiology Fondation Hôpital Saint Joseph

Paris, France

M. Rodallec

Department of Radiology

Centre Cardiologique du Nord Saint Denis, France

$\mathrm{X}$. Leclerc

Department of Neuroradiology Hôpital Roger Salengro Lille, France

R. Blanc

Fondation Ophtalmologique Adolphe de Rothschild

Paris, France

A. Rahmouni

Department of Radiology Hôpital Henri Mondor

Créteil, France

http://dx.doi.org/10.3174/ajnr.A2875 